\title{
Frequency Domain System Identification with Instrumental Variable based Algorithm
}

\author{
Tomas McKelvey \\ Department of Electrical Engineering \\ Linkping University, S-581 83 Linkping, Sweden \\ WWW: http://www.control.isy.liu.se \\ Email: tomas@isy.liu.se
}

March 1999

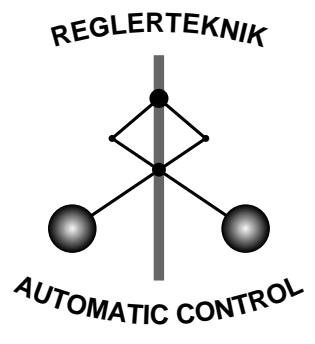

LINKÖPING

Report no.: LiTH-ISY-R-2135

Proc. 16th Biennal Conference on Mechanical Vibaration and Noise, 1997

Technical reports from the Automatic Control group in Linkping are available by anonymous ftp at the address ftp.control.isy.liu.se. This report is contained in the pdf-file 2135.pdf. 


\title{
DETC97/VIB-4252
}

\section{FREQUENCY DOMAIN SYSTEM IDENTIFICATION WITH INSTRUMENTAL VARIABLE BASED SUBSPACE ALGORITHM}

\author{
Tomas McKelvey \\ Automatic Control \\ Department of Electrical Engineering \\ Linköping University \\ S-58183 Linkp̈ing, Sweden \\ Email: tomas@isy.liu.se
}

\begin{abstract}
In this paper we discuss how the time domain subspace based identification algorithms can be modified in order to be applicable when the primary measurements are given as samples of the Fourier transform of the input and output signals or alternatively samples of the transfer function. An instrumental variable (IV) based subspace algorithm is presented. We show that this method is consistent if a certain rank constraint is satisfied and the frequency domain noise is zero mean with bounded covariances. An example is presented which illuminates the theoretical discussion.
\end{abstract}

Keywords: system identification, state-space models, subspace methods, numerical methods, stochastic analysis, frequency domain, approximation

\section{INTRODUCTION}

Methods which identify state-space models by means of geometrical properties of the input and output sequences are commonly known as subspace methods and have received much attention in the literature. The early subspace identification methods (De Moor and Vandewalle 1987; Moonen, De Moor, Vandenberghe, and Vandewalle 1989; Verhaegen 1991) focused on the deterministic systems with errors represented at the outputs. By extending these methods, consistent algorithms have been obtained when the errors are described by colored noise (Van Overschee and De Moor 1994; Verhaegen 1993; Verhaegen 1994). One of the advantages with subspace methods is the absence of a parametric iterative optimization step. In classical prediction error minimization (Ljung 1987), such a step is nec- essary for most model structures. A second advantage is that the identification of multivariable systems is just as simple as for scalar systems. Particularly one do not have to deal with the difficult parametrization issue of multivariable systems since no explicit parametrization is needed. An overview of time domain subspace methods is given by Viberg (1995).

In this paper we consider the case when data is given in the frequency domain, i.e. when samples of the Fourier transform of the input and output signals are the primary measurements. In a number of applications, particularly when modeling flexible structures, it is common to fit models in the frequency domain (Schoukens and Pintelon 1991; Ljung 1993). A few subspace based algorithms formulated in the frequency domain has appeared recently. A frequency domain version by Liu and Skelton (1993) has been described in (Liu, Jacques, and Miller 1994). Two related algorithms has been presented in (McKelvey, Akçay, and Ljung 1996). These algorithms use a discrete time formulation. In (Van Overschee and De Moor 1996) a subspace based algorithm is presented which is based on a continuoustime frequency domain formulation. One drawback of all these methods is that they are only consistent if the second order noise statistics are known. A method is consistent if the algorithm converges to the true system as the number of noisy frequency samples tend to infinity, i.e. the frequency grid become denser and denser.

A different route is followed in in (McKelvey and Akçay 1994; McKelvey, Akçay, and Ljung 1996) where an algorithm which is based on the inverse discrete Fourier trans- 
form is presented. This algorithm is consistent for a large class of systems but requires samples of the frequency response of the system at equidistant frequencies covering the whole frequency axis $(0-\pi)$.

The topic of this paper is to introduce a subspace based identification algorithm which is consistent also for the case of unknown second order noise statistics which is applicable for frequency data sampled at arbitrary frequencies. We do this by the technique of instrumental variables (Söderström and Stoica 1989). A similar technique has previously been used for a time domain algorithm called PI-MOESP (Verhaegen 1993).

In the sequel we will only consider the case of identifying a discrete time system from samples of the discrete time Fourier transform of the inputs and outputs. The same algorithms can also be used to identify continuous-time systems from continuous time data by adding a bilinear transformation step. For more details we refer to (McKelvey, Akçay, and Ljung 1996).

\section{PRELIMINARIES}

Consider a stable time-invariant discrete time linear system of finite order $n$ in state-space form

$$
\begin{aligned}
x(k+1) & =A x(k)+B u(k) \\
y(k) & =C x(k)+D u(k)+v(k)
\end{aligned}
$$

where $u(k) \in \mathbb{R}^{m}$ is the input vector, $y(k) \in \mathbb{R}^{p}$ the output vector and $x(k) \in \mathbb{R}^{n}$ is the state vector. The noise term $v(k) \in \mathbb{R}^{p}$ is assumed to be independent of the input sequence $u(t)$. Here the time index $k$ denotes normalized time. Hence $y(k)$ denotes the sample of the output signal $y(t)$ at time instant $t=k T$ where $T$ denotes the sample time. We also assume that the state-space realization (1) is minimal which implies both observability and controllability (Kailath 1980). A system with this type of noise model is commonly known as output-error models (Ljung 1987). Note that all such pairs (1) describing the same input/output behavior of the system are equivalent under a non-singular similarity transformation $T \in \mathbb{R}^{n \times n}$ (Kailath $1980)$, i.e the matrices $\left(T^{-1} A T, T^{-1} B, C T, D\right)$ will be an equivalent state-space realization. The discrete time Fourier transform $\mathcal{F}$ of a sequence $f(k)$ is defined as

$$
\mathcal{F} f(k)=F(\omega)=\sum_{k=-\infty}^{\infty} f(k) e^{-j \omega k}
$$

where $j=\sqrt{-1}$. Applying the Fourier transform to (1) gives

$$
\begin{aligned}
e^{j \omega} X(\omega) & =A X(\omega)+B U(\omega) \\
Y(\omega) & =C X(\omega)+D U(\omega)+V(\omega)
\end{aligned}
$$

where $Y(\omega), U(\omega), V(\omega)$ and $X(\omega)$ are the transformed output, input, noise and state respectively. By eliminating the state from (3) we obtain

$$
Y(\omega)=G\left(e^{j \omega}\right) U(\omega)+V(\omega)
$$

where

$$
G(z)=D+C(z I-A)^{-1} B
$$

is known as the transfer function of the linear system.

\section{The Identification Problem}

Given samples of the discrete time Fourier transform of the input signal $U(\omega)$ and output signal $Y(\omega)$ at $N$ arbitrary frequency points $\omega_{k}$; find a state-space model of the form (1) which well approximates the data. Particularly we will focus on deriving an algorithm which is consistent.

\section{SUBSPACE IDENTIFICATION}

In this section we will derive the basic relations which the subspace based identification algorithm rely upon and present an consistent identification algorithm.

\section{The Basic Relations}

By introducing the vector

$$
W(\omega)=\left[1 e^{j \omega} e^{j 2 \omega} \cdots e^{j \omega(q-1)}\right]^{T}
$$

the extended observability matrix with $q$ block rows

$$
\mathcal{O}_{q}=\left[\begin{array}{c}
C \\
C A \\
\vdots \\
C A^{q-1}
\end{array}\right]
$$

and the lower triangular Toeplitz matrix

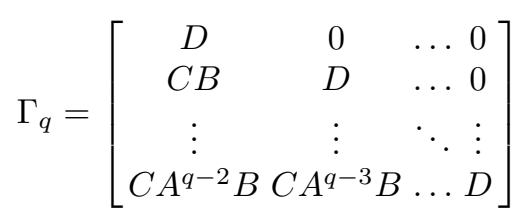


we will by recursive use of (3) obtain

$W(\omega) \otimes Y(\omega)=\mathcal{O}_{q} X(\omega)+\Gamma_{q} W(\omega) \otimes U(\omega)+W(\omega) \otimes V(\omega)$

where $\otimes$ denote the Kronecker product (Horn and Johnson 1991). The extended observability matrix $\mathcal{O}_{q}$ has a rank equal to the system order $n$ if $q \geq n$ since the system $(A, B, C, D)$ is minimal.

If $N$ samples of the transforms are known we can collect all data into one matrix equation. By introducing the additional matrices

$$
\begin{gathered}
\mathbf{W}_{q, N, p}=\left[W\left(\omega_{1}\right) W\left(\omega_{2}\right) \cdots W\left(\omega_{N}\right)\right] \otimes I_{p} \\
\mathbf{Y}_{q, N}=\frac{1}{\sqrt{N}} \mathbf{W}_{q, N, p} \operatorname{diag} Y\left(\omega_{1}\right), \ldots, Y\left(\omega_{N}\right) \in \mathbb{C}^{q p \times N}, \\
\mathbf{U}_{q, N}=\frac{1}{\sqrt{N}} \mathbf{W}_{q, N, m} \operatorname{diag} U\left(\omega_{1}\right), \ldots, U\left(\omega_{N}\right) \in \mathbb{C}^{q m \times N}, \\
\mathbf{V}_{q, N}=\frac{1}{\sqrt{N}} \mathbf{W}_{q, N, p} \operatorname{diag} V\left(\omega_{1}\right), \ldots, V\left(\omega_{N}\right) \in \mathbb{C}^{q p \times N} \\
\mathbf{X}_{N}=\frac{1}{\sqrt{N}}\left(X\left(\omega_{1}\right), X\left(\omega_{2}\right), \cdots, X\left(\omega_{N}\right),\right) \in \mathbb{C}^{n \times N}
\end{gathered}
$$

and using (9) we arrive at the matrix equation

$$
\mathbf{Y}_{q, N}=\mathcal{O}_{q} \mathbf{X}_{N}+\Gamma_{q} \mathbf{U}_{q, N}+\mathbf{V}_{q, N}
$$

As the number of frequency samples grows the number of (block)-columns in this matrix equation also grows. The normalization with $\frac{1}{\sqrt{N}}$ ensures that the norm of the matrix stays bounded as the number of frequencies (columns) tends to infinity. The number of (block)-rows $q$ is up to the user to choose but must be larger than the upper bound of the model orders which will be considered.

Remark 1. If instead samples of the transfer function $G\left(e^{j \omega_{k}}\right)$ are given a similar matrix expression as (14) can be derived (McKelvey, Akçay, and Ljung 1996). Consequently the identification algorithm outlined below can also be used for this problem.

\section{The Main Steps of the Identification}

The identification scheme we employ to find an statespace model $(\hat{A}, \hat{B}, \hat{C}, \hat{D})$ is based on a two step procedure. First the relation (14) is used to consistently determine a matrix $\hat{\mathcal{O}}_{q}$ with a range space equal to the extended observability matrix $\mathcal{O}_{q}$. From $\hat{\mathcal{O}}_{q}$ it is straight forward to derive $\hat{A}$ and $\hat{C}$ as is well known from the time domain subspace methods (Viberg 1994). In the second step $\hat{B}$ and $\hat{D}$ are determined by minimizing the Frobenius norm of the error

$$
\hat{B}, \hat{D}=\arg \min _{B, D} V_{N}(B, D)
$$

$$
V_{N}(B, D)=\sum_{k=1}^{N}\left\|Y\left(\omega_{k}\right)-\hat{Y}\left(\omega_{k}, B, D\right) U\left(\omega_{k}\right)\right\|_{F}^{2}
$$

where

$$
\hat{Y}\left(\omega_{k}, B, D\right)=\left[D-\hat{C}\left(e^{j \omega_{k}} I-\hat{A}\right)^{-1} B\right] U\left(\omega_{k}\right)
$$

which has an analytical solution since the transfer function $G$ is a linear function of both $B$ and $D$ assuming $\hat{A}$ and $\hat{C}$ are fixed.

\section{The Basic Projection Method}

First consider the noise free case $\mathbf{V}_{q, N}=0$ and we restate the basic projection method (De Moor and Vandewalle 1987; De Moor 1988) in the frequency domain. In (14) the term $\Gamma_{q} \mathbf{U}_{q, N}$ can be removed by the use of $\boldsymbol{\Pi}_{N}^{\perp}$ which is the orthogonal projection onto the null-space of $\mathbf{U}_{q, N}$,

$$
\boldsymbol{\Pi}_{N}^{\perp}=I-\mathbf{U}_{q, N}^{*}\left(\mathbf{U}_{q, N} \mathbf{U}_{q, N}^{*}\right)^{-1} \mathbf{U}_{q, N}
$$

here $\mathbf{U}_{q, N}^{*}$ denotes the complex conjugate and transpose of the matrix $\mathbf{U}_{q, N}$. The inverse in 17 will exist if the system is sufficiently excited by the input. Particularly we require that for some positive constants $c$ and $N_{0}$ that

$$
\mathbf{U}_{q, N} \mathbf{U}_{q, N}^{*}>c I, \quad \forall N>N_{0}
$$

Since

$$
\mathbf{U}_{q, N} \boldsymbol{\Pi}_{N}^{\perp}=0
$$

the effect of the input will be removed and we obtain

$$
\mathbf{Y}_{q, N} \boldsymbol{\Pi}_{N}^{\perp}=\mathcal{O}_{q} \mathbf{X}_{N} \boldsymbol{\Pi}_{N}^{\perp}
$$


Provided

$$
\operatorname{rank}\left(\mathbf{X}_{N} \boldsymbol{\Pi}_{N}^{\perp}\right)=n
$$

$\mathbf{Y}_{q, N} \boldsymbol{\Pi}_{N}^{\perp}$ and $\mathcal{O}_{q}$ will span the same column space. The mild conditions required for (20) to hold can be found in (McKelvey, Akçay, and Ljung 1996).

The state-space matrices $(A, B, C, D)$ and thus also the extended observability matrices are usually real matrices but $\mathbf{Y}_{q, N} \boldsymbol{\Pi}_{N}^{\perp}$ is a complex matrix. The real space can be recovered by using both the real part and the imaginary part in a singular value decomposition (Horn and Johnson 1985)

$$
\left[\operatorname{Re}\left(\mathbf{Y}_{q, N} \boldsymbol{\Pi}_{N}^{\perp}\right) \operatorname{Im}\left(\mathbf{Y}_{q, N} \boldsymbol{\Pi}_{N}^{\perp}\right)\right]=\left[U_{s} U_{o}\right]\left[\begin{array}{cc}
\Sigma_{s} & 0 \\
0 & \Sigma_{o}
\end{array}\right]\left[\begin{array}{c}
V_{s}^{T} \\
V_{o}^{T}
\end{array}\right]
$$

where $U_{s} \in \mathbb{R}^{q p \times n}$ contains the $n$ principal singular vectors and the diagonal matrix $\Sigma_{s}$ the corresponding singular values. In the noise free case $\Sigma_{o}=0$ and there will exist a nonsingular matrix $T \in \mathbb{R}^{n \times n}$ such that

$$
\mathcal{O}_{q}=U_{s} T
$$

This shows that $U_{s}$ is an extended observability matrix $\hat{\mathcal{O}}_{q}$ of the original system in some realization. By the shift structure of the observability matrix we can proceed to calculate $A$ and $C$ as

$$
\begin{gathered}
\hat{A}=\left(J_{1} U_{s}\right)^{\dagger} J_{2} U_{s} \\
\hat{C}=J_{3} U_{s}
\end{gathered}
$$

where $J_{i}$ are the selection matrices defined by

$$
\begin{aligned}
& J_{1}=\left(\begin{array}{ll}
I_{(q-1) p} & 0_{(q-1) p \times p}
\end{array}\right), \quad J_{2}=\left(0_{(q-1) p \times p} I_{(q-1) p}\right) \\
& J_{3}=\left(\begin{array}{ll}
I_{p} & 0_{p \times(q-1) p}
\end{array}\right)
\end{aligned}
$$

and $I_{i}$ denotes the $i \times i$ identity matrix, $0_{i \times j}$ denotes the $i \times j$ zero matrix and $X^{\dagger}=\left(X^{T} X\right)^{-1} X^{T}$ denotes the MoorePenrose pseudo-inverse of the full rank matrix $X$. With the knowledge of $\hat{A}$ and $\hat{C}, \hat{B}$ and $\hat{D}$ are easily determined from (15).
Effective Implementation A most effective way of forming the matrix $\mathbf{Y}_{q, N} \boldsymbol{\Pi}_{N}^{\perp}$ is by use of the QR factorization of the matrix (Verhaegen 1991)

$$
\left(\begin{array}{c}
\mathbf{U}_{q, N} \\
\mathbf{Y}_{q, N}
\end{array}\right)=\left(\begin{array}{cc}
\mathbf{R}_{11} & 0 \\
\mathbf{R}_{21} & \mathbf{R}_{22}
\end{array}\right)\left(\begin{array}{l}
\mathbf{Q}_{1}^{*} \\
\mathbf{Q}_{2}^{*}
\end{array}\right)
$$

Straight forward calculations reveal that

$$
\mathbf{Y}_{q, N} \boldsymbol{\Pi}_{N}^{\perp}=\mathbf{R}_{22} \mathbf{Q}_{2}^{*}
$$

and the column space of $\mathbf{R}_{22}$ is equal to the column space of $\mathbf{Y}_{q, N} \boldsymbol{\Pi}_{N}^{\perp}$ and it suffices to use $\mathbf{R}_{22}$ in the SVD (21).

Consistency Issues As we have seen the basic projection algorithm will estimate a state-space model which is similar to the original realization in the noise free case. If we now let the noise term $N(\omega)$ be a zero mean complex random variable the issue of consistency becomes important. Does the estimate converge to the true system as $N$, the number of data, tends to infinity? Consistency of the basic projection algorithm and the related algorithm (Liu, Jacques, and Miller 1994) has been investigated in (McKelvey, Akçay, and Ljung 1996). The basic projection algorithm is consistent if the frequency data is given at equidistant frequencies covering the entire unit circle and the noise $N\left(\omega_{k}\right)$ is zero mean and have equal covariance proportional to the identity matrix for all frequencies. The uniform covariance requirement for all frequencies and the need for an equidistant frequency grid limits the practical use of the basic projection algorithm. If the covariance of the noise is known the basic algorithm can be extended with certain weighting matrices in order to obtain a consistent algorithm. See (McKelvey, Akçay, and Ljung 1996; Van Overschee and De Moor 1996) for further details.

\section{Instrumental Variable Techniques}

The strict noise properties required in order to guarantee consistency is a severe drawback for the basic projection method. The origin of the problem stems from the fact that the noise influence does not disappear from the estimate but is required to converge to an identity matrix. What we would like is to find some instruments which are uncorrelated with the noise but preserves the rank condition (20). A similar technique as in the time domain instrumental variable algorithms in (Verhaegen 1993; Verhaegen 1994) will here be adopted to yield a frequency domain instrumental variable method. 
Let us assume we can find a matrix $\mathbf{U}_{I V, \alpha, N} \in \mathbb{C}^{m \alpha \times N}$, called the instruments, with the following two properties

$$
\operatorname{rank}\left(\mathbf{X}_{N} \boldsymbol{\Pi}_{N}^{\perp} \mathbf{U}_{I V, \alpha, N}^{*}\right)=n, \quad \forall N>N_{0}
$$

and w.p. 1 .

$$
\left\|\mathbf{V}_{q, N} \boldsymbol{\Pi}_{N}^{\perp} \mathbf{U}_{I V, \alpha, N}^{*}\right\|_{F} \rightarrow 0, \quad \text { as } N \rightarrow \infty
$$

In other words we want to find a matrix which is correlated with the output matrix and uncorrelated with the noise matrix.

One possible choice is to use a "continuation" of the matrix $\mathbf{U}_{q, N}$ according to

$$
\left[\begin{array}{c}
\mathbf{U}_{q, N} \\
\mathbf{U}_{I V, \alpha, N}
\end{array}\right]=\mathbf{U}_{q+\alpha, N}
$$

where $\mathbf{U}_{q+\alpha, N}$ is defined according to (12). Such a matrix is independent of $V(\omega)$ since by assumption the noise is independent of the input. Furthermore, by construction it is obvious that the row spaces of $\mathbf{U}_{q, N}$ and $\mathbf{U}_{I V, \alpha, N}$ do not intersect for $m(q+\alpha) \leq N$. Consequently we have

$$
\operatorname{rank}\left(\boldsymbol{\Pi}_{N}^{\perp} \mathbf{U}_{I V, \alpha, N}^{*}\right)=m \alpha
$$

It is still an open question if (27) is satisfied for this particular choice of $\mathbf{U}_{I V, \alpha, N}$. However, experience indicates that (27) is the generic case.

Assume $V\left(\omega_{k}\right)$ to be zero mean independent random variables with uniformly bounded second moments

$$
E V\left(\omega_{k}\right) V\left(\omega_{k}\right)^{*}=R\left(\omega_{k}\right) \leq R, \quad \forall \omega_{k}
$$

where $E$ denote the expectation operator. Then the following relation follows from a standard limit result with probability one (w.p. 1) (Chung 1974, Theorem 5.1.2)

$$
\lim _{N \rightarrow \infty} \mathbf{V}_{q, N} \boldsymbol{\Pi}_{N}^{\perp} \mathbf{U}_{I V, \alpha, N}^{*}=0
$$

Thus, if the rank condition (27) is fulfilled the $n$ principal left singular vectors of

$$
\mathbf{Y}_{q, N} \boldsymbol{\Pi}_{N}^{\perp} \mathbf{U}_{I V, \alpha, N}^{*}
$$

will constitute a strongly consistent estimate of the range space of the extended observability matrix (7).
Implementation Just as for the basic projection algorithm an efficient implementation involves an $Q R$ factorization of the data matrices. By following (Verhaegen 1993) we form the QR factorization

$$
\left[\begin{array}{c}
\mathbf{U}_{q, N} \\
\mathbf{U}_{I V, \alpha, N} \\
\mathbf{Y}_{q, N}
\end{array}\right]=\left[\begin{array}{ccc}
\mathbf{R}_{11} & 0 & 0 \\
\mathbf{R}_{21} & \mathbf{R}_{22} & 0 \\
\mathbf{R}_{31} & \mathbf{R}_{32} & \mathbf{R}_{32}
\end{array}\right]\left[\begin{array}{l}
\mathbf{Q}_{1}^{*} \\
\mathbf{Q}_{2}^{*} \\
\mathbf{Q}_{3}^{*}
\end{array}\right]
$$

By using (30) it is straightforward to show that

$$
\mathbf{Y}_{q, N} \boldsymbol{\Pi}_{N}^{\perp} \mathbf{U}_{I V, \alpha, N}^{*}=\mathbf{R}_{32} \mathbf{R}_{22}^{*}
$$

and we will use $\mathbf{R}_{32}$ in a SVD to estimate the range space of the observability matrix since $\mathbf{R}_{22}$ is of full rank whenever $\mathbf{U}_{q+\alpha, N}$ has full rank. The observability range space is thus extracted as $U_{s} \in \mathbb{R}^{q p \times n}$ from

$$
\left[\operatorname{Re}\left(\mathbf{R}_{32}\right) \operatorname{Im}\left(\mathbf{R}_{32}\right)\right]=\left[\begin{array}{ll}
U_{s} & U_{o}
\end{array}\right]\left[\begin{array}{cc}
\Sigma_{s} & 0 \\
0 & \Sigma_{o}
\end{array}\right]\left[\begin{array}{l}
V_{s}^{*} \\
V_{o}^{*}
\end{array}\right]
$$

Notice that the orthogonal matrix $\mathbf{Q}$ in (30) is not needed in the estimation and the QR factorization constitutes a considerable data reduction since the size of $\mathbf{R}_{32} \in \mathbb{C}^{q p \times \alpha m}$ is independent of the number of data samples $N$. As before we use $U_{s}$ as the estimate of the extended observability matrix and determine $\hat{A}$ and $\hat{C}$ according to (22) and (23) while $\hat{B}$ and $\hat{D}$ are determined from (15). By using the $\hat{A}$ and $\hat{C}$ from the consistent estimates of the observability range space the solution of $\hat{B}$ and $\hat{D}$ from (15) is a linear function of the output Fourier transforms $Y\left(\omega_{k}\right)$ and hence also in the noise. By similar arguments as before this shows that $\hat{B}$ and $\hat{D}$ will be also will be consistently estimated, se also (McKelvey, Akçay, and Ljung 1996).

We summarize this discussion in the form of an identification algorithm.

\section{Algorithm 1.}

1. Determine an upper bound $\bar{n}$ of the model order. Select the size variables $q$ and $\alpha$ such that $q>\bar{n}$ and $\alpha \geq \bar{n}$.

2. Form the matrices $\mathbf{Y}_{q, N}$ (11), $\mathbf{U}_{q+\alpha, N}$ (12) and partition it as (29)

3. Calculate the $Q R$ factorization

$$
\left[\begin{array}{c}
\mathbf{U}_{q, N} \\
\mathbf{U}_{I V, \alpha, N} \\
\mathbf{Y}_{q, N}
\end{array}\right]=\left[\begin{array}{ccc}
\mathbf{R}_{11} & 0 & 0 \\
\mathbf{R}_{21} & \mathbf{R}_{22} & 0 \\
\mathbf{R}_{31} & \mathbf{R}_{32} & \mathbf{R}_{32}
\end{array}\right]\left[\begin{array}{c}
\mathbf{Q}_{1}^{*} \\
\mathbf{Q}_{2}^{*} \\
\mathbf{Q}_{3}^{*}
\end{array}\right]
$$


4. Calculate the SVD of $\mathbf{R}_{32}$ and determine the model order $n$ by inspecting the singular values. Partition the $S V D$ as

$$
\left[\operatorname{Re}\left(\mathbf{R}_{32}\right) \operatorname{Im}\left(\mathbf{R}_{32}\right)\right]=U_{s} \Sigma_{s} V_{s}^{T}+U_{o} \Sigma_{o} V_{o}^{T}
$$

were $U_{s}$ contain the left singular vectors of the $n$ dominating singular values.

5. Determine $\hat{A}$ and $\hat{C}$ :

$$
\hat{A}=\left(J_{1} U_{s}\right)^{\dagger} J_{2} U_{s}, \quad \hat{C}=J_{3} U_{s}
$$

6. Solve the least-squares problem for $\hat{B}$ and $\hat{D}$ according to (15).

We also summarize the theoretical discussion in the following theorem.

Theorem 1. Assume that the following conditions are satisfied:

(i) The frequency data is generated by a stable linear system $G(z)$ of order $n$.

(ii) There exist positive constants $c$ and $N_{0}$ such that

$$
\mathbf{U}_{q+\alpha, N} \mathbf{U}_{q+\alpha, N}^{*}>c I, \quad \forall N>N_{0}
$$

(iii) $\operatorname{rank}\left(\mathbf{X}_{N} \boldsymbol{\Pi}_{N}^{\perp} \mathbf{U}_{I V, \alpha, N}^{*}\right)=n$

(iv) $V\left(\omega_{k}\right)$ are zero mean independent random variables with bounded covariances

$$
E V\left(\omega_{k}\right) V\left(\omega_{k}\right)^{*}=R_{k} \leq R<\infty, \quad \forall \omega_{k}
$$

Let $\hat{G}(z)$ be the resulting transfer function when applying Algorithm 1. Then

$$
\lim _{N \rightarrow \infty} \sup _{z=1}\|G(z)-\hat{G}(z)\|_{F}=0, \quad \text { w.p } 1
$$

Remark 2. From the construction of $\mathbf{U}_{q, N}$ (12) we notice that in the single input case $m=1$ the rank condition (ii) is equivalent to require that at least $q+\alpha$ samples of $U\left(\omega_{k}\right)$ are non-zero. For the multivariable case the condition is somewhat more involved.

\section{ILLUSTRATING EXAMPLE}

This section describes an identification example based on simulated data. From the results of the example we will clearly see the limits of the basic projection algorithm when faced with data which do not comply with the assumptions needed for consistence. On the other hand the instrumental variable algorithm we will experience to perform as predicted by the consistency result of Theorem 1 .

\section{Experimental Setup}

Let the true system $G(z)$ be a fourth order system with an output error noise model $H(z)$. In the frequency domain we thus assume

$$
Y(\omega)=G\left(e^{j \omega}\right) U(\omega)+H\left(e^{j \omega}\right) E(\omega)
$$

where $Y(\omega), U(\omega)$ and $E(\omega)$ are the Fourier transform of the time domain quantities; outputs $y(t)$, inputs $u(t)$ and innovations $e(t)$. The system $G(z)$ is given by

$$
G(z)=C(z I-A)^{-1} B+D
$$

with

$$
\begin{aligned}
& A=\left(\begin{array}{cccc}
0.8876 & 0.4494 & 0 & 0 \\
-0.4494 & 0.7978 & 0 & 0 \\
0 & 0 & -0.6129 & 0.0645 \\
0 & 0 & -6.4516 & -0.7419
\end{array}\right), \quad B=\left(\begin{array}{c}
0.2247 \\
0.8989 \\
0.0323 \\
0.1290
\end{array}\right) \\
& C=\left(\begin{array}{llll}
0.4719 & 0.1124 & 9.6774 & 1.6129
\end{array}\right), \quad D=0.9626 .
\end{aligned}
$$

The noise transfer function is of second order and is given by

$$
H(z)=C_{n}\left(z I-A_{n}\right)^{-1} B_{n}+D_{n}
$$

with

$$
\begin{array}{ll}
A_{n}=\left[\begin{array}{cc}
0.6296 & 0.0741 \\
-7.4074 & 0.4815
\end{array}\right], & B_{n}=\left[\begin{array}{l}
0.0370 \\
0.7407
\end{array}\right] \\
C_{n}=\left[\begin{array}{ll}
1.6300 & 0.0740
\end{array}\right], & D_{n}=0.2000 .
\end{array}
$$

The Fourier transform of the noise $E(\omega)$ is modeled as a complex Gaussian distributed random variable with unit variance and is assumed to be independent over different frequencies. In the output error formalism we obtain the output error as

$$
V(\omega)=H\left(e^{j \omega}\right) E(\omega)
$$

which thus is a complex Gaussian random variable with frequency dependent variance equal to $\left|H\left(e^{j \omega}\right)\right|^{2}$. The Fourier 


\begin{tabular}{|c|cc|}
\hline \multirow{2}{*}{$\mathrm{N}$} & \multicolumn{2}{|c|}{ Average $\|G-\hat{G}\|_{\infty}$} \\
\cline { 2 - 3 } & Proj. Alg. & IV Alg. \\
\hline 30 & 1.7886 & 1.0425 \\
50 & 1.3829 & 0.7737 \\
100 & 1.2867 & 0.5078 \\
200 & 1.2638 & 0.3751 \\
400 & 1.2378 & 0.2550 \\
\hline
\end{tabular}

Table 1. Monte Carlos simulations comparing the basic projection algorithm and the IV algorithm. The estimation error decreases for an increasing amount of identification data which is predicted from Theorem 1. The projection algorithms fails to capture the true system which show that the assumption of evenly spaced frequencies and equal covariances are essentially necessary for the projection algorithm to be consistent.

transform of the input signal is defined to be $U(\omega)=1, \forall \omega$, i.e. all frequencies are equally excited.

To examine the consistency properties of the basic projection algorithm and the IV algorithm we perform Monte Carlo simulations estimating the system given samples of $U(\omega)$ and $Y(\omega)$ using different noise realizations of $E(\omega)$ and an increasing number of samples of the transforms. The frequency grid will be logarithmically spaced between $\omega_{1}=0.3$ and $\omega_{N}=\pi$. Data lengths of 30, 50, 100, 200 and 400 frequency samples will be used. For each data length 100 different noise realizations are generated and both algorithms estimate 100 models. To assess the quality of the resulting model the max norm of the estimation error

$$
\|G(z)-\hat{G}(z)\|_{\infty}=\sup _{|z|=1}\|G(z)-\hat{G}(z)\|_{F}
$$

is determined for each estimated model and averaged over the 100 estimated models.

\section{Estimation Results}

As expected from the analysis the quality of the estimates from the instrumental variable algorithm (IV) improves as the number of samples of the Fourier transform increases. In Table 1 the averaged maximum identification error is presented. The results clearly indicate that the basic projection algorithm is not consistent for these data. We have in this example violated the requirement of equally spaced frequencies and equal noise covariances required for consistency of the basic projection algorithm (McKelvey and Akçay 1994) and by judging from the example these requirements seems to be essentially necessary for consistency.

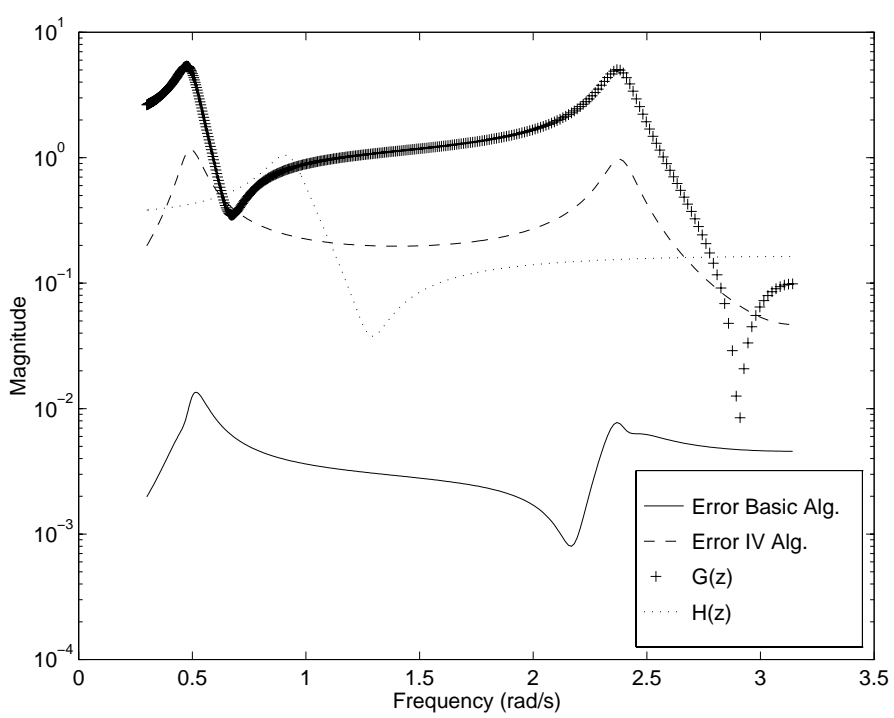

Figure 1. Result from Monte Carlo simulations using data length $N=400$. The true transfer function $G(z)$ is depicted as "+" and the noise transfer function $H(z)$ is shown as the dotted line. The absolute value of the mean transfer function errors calculated over 100 estimated models are shown as a solid line for the IV method and as a dashed line for the basic projection method.

\section{CONCLUSIONS}

In this paper we have derived an instrumental variable subspace algorithm for the case when the primary measurements are given as samples of the Fourier transform of the input and output signals. We have shown that the method is consistent if a certain rank constraint is satisfied and the frequency domain noise is zero mean and have bounded covariance. An example is presented which illuminate the theoretical discussion.

\section{ACKNOWLEDGMENT}

This work was supported in part by the Swedish Research Council for Engineering Sciences (TFR), which is gratefully acknowledged.

\section{References}

Chung, K. L. (1974). A Course in Probability Theory. Academic Press, San Diego, CA.

De Moor, B. (1988, June). Mathematical Concepts and Techniques for Modeling of Static and Dynamic Systems. Ph. D. thesis, Katholieke Universiteit Leuven, 
Kard. Mercierlaan 94, 3001 Leuven (Heverlee), Belgium.

De Moor, B. and J. Vandewalle (1987). A geometrical strategy for the identification of state space models of linear multivariable systems with singular value decomposition. In Proc. of the 3rd International Symposium on Applications of Multivariable System Techniques, April 13-15, Plymouth, UK, pp. 59-69.

Horn, R. A. and C. R. Johnson (1985). Matrix Analysis. Cambridge University Press, Cambridge, NY.

Horn, R. A. and C. R. Johnson (1991). Topics In Matrix Analysis. Cambridge University Press, Cambridge, NY.

Kailath, T. (1980). Linear Systems. Englewood Cliffs, New Jersey: Prentice-Hall.

Liu, K., R. N. Jacques, and D. W. Miller (1994, June). Frequency domain structural system identification by observability range space extraction. In Proc. of the American Control Conference, Baltimore, Maryland, Volume 1, pp. 107-111.

Liu, K. and R. E. Skelton (1993). Q-markov covariance equivalent realization and its application to flexible structure identification. AIAA Journal of Guidance, Control and Dynamics 16(2), 308-319.

Ljung, L. (1987). System Identification: Theory for the User. Englewood Cliffs, New Jersey: Prentice-Hall.

Ljung, L. (1993, December). Some results on identifying linear systems using frequency domain data. In Proc. 32nd IEEE Conference on Decision and Control, San Antonio, Texas, pp. 3534-3538.

McKelvey, T. and H. Akçay (1994, December). An efficient frequency domain state-space identification algorithm: Robustness and stochastic analysis. In Proc. 33rd IEEE Conference on Decision and Control, Lake Buena Vista, Florida, pp. 3348-3353.

McKelvey, T., H. Akçay, and L. Ljung (1996, July). Subspace-based multivariable system identification from frequency response data. IEEE Trans. on Automatic Control 41(7), 960-979.

Moonen, M., B. De Moor, L. Vandenberghe, and J. Vandewalle (1989). On- and off-line identification of linear state-space models. Int. J. Control 49(1), 219-232.

Schoukens, J. and R. Pintelon (1991). Identification of Linear Systems: a Practical Guideline to Accurate Modeling. Pergamon Press, London, UK.

Söderström, T. and P. Stoica (1989). System Identification. Hemel Hempstead, Hertfordshire: Prentice-Hall International.

Van Overschee, P. and B. De Moor (1994). N4SID: Subspace algorithms for the identification of combined deterministic-stochastic systems. Automatica 30(1), 75-93.
Van Overschee, P. and B. De Moor (1996, July). Continuous-time frequency domain subspace system identification. Signal Processing, EURASIP 52(2), 179-194.

Verhaegen, M. (1991, July). A novel non-iterative MIMO state space model identification technique. In Proc. 9th IFAC/IFORS Symp. on Identification and System parameter estimation, Budapest, Hungary, pp. 14531458.

Verhaegen, M. (1993). Subspace model identification, Part III: Analysis of the ordinary output-error state space model identification algorithm. Int. J. Control 58, 555-586.

Verhaegen, M. (1994). Identification of the deterministic part of MIMO state space models given in innovations form from input-output data. Automatica 30(1), 6174.

Viberg, M. (1994). Subspace methods in system identification. In 10th IFAC Symposium on System Identification, Volume 1, Copenhagen, Denmark, pp. 1-12. IFAC.

Viberg, M. (1995). Subspace-based methods for the identification of linear time-invariant systems. Automatica 31(12), 1835-1851. 